\title{
Serious complications and recurrences after pelvic organ prolapse surgery for 2309 women in the VIGI-MESH registry
}

Xavier Fritel ${ }^{1}$, Renaud de Tayrac ${ }^{2}$, Joe de Keizer ${ }^{3}$, Sandrine Campagne-Loiseau ${ }^{4}$, Michel Cosson $^{5}$, Philippe Ferry ${ }^{6}$, Xavier Deffieux ${ }^{7}$, Jean-Philippe Lucot ${ }^{8}$, Laurent Wagner ${ }^{9}$, Philippe Debodinance ${ }^{10}$, Christian Saussine ${ }^{11}$, Anne-Cécile Pizzoferrato ${ }^{12}$, Caroline Carlier-Guérin $^{13}$, Thibault Thubert ${ }^{14}$, Laure Panel ${ }^{15}$, Pierre-Olivier Bosset ${ }^{16}$, Elvis Nkounkou ${ }^{17}$, Rajeev Ramanah ${ }^{18}$, Thomas Boisramé ${ }^{11}$, Thomas Charles $^{19}$, Cyril Raiffort ${ }^{20}$, Amélie Charvériat ${ }^{1}$, Stéphanie Ragot ${ }^{3}$, and Arnaud Fauconnier ${ }^{21}$

${ }^{1} \mathrm{CHU}$ de Poitiers

${ }^{2}$ CHU Carémeau

${ }^{3}$ Université de Poitiers UFR Médecine et Pharmacie

${ }^{4}$ CHU Estaing

${ }^{5}$ Hopital Jeanne de Flandre, Centre Hospitalier Regional Universitaire de Lille

${ }^{6} \mathrm{CH}$ La Rochelle

${ }^{7} \mathrm{CHU}$ Antoine Beclere

${ }^{8}$ Hôpital Saint-Vincent de Paul

${ }^{9}$ Medical University Nimes

${ }^{10} \mathrm{CH}$ Dunkerque

${ }^{11}$ CHU Strasbourg

${ }^{12} \mathrm{CHU}$ Caen

${ }^{13} \mathrm{CH}$ de Châtellerault

${ }^{14}$ Centre Hospitalier Universitaire de Nantes

${ }^{15}$ Clinique Beau Soleil

${ }^{16}$ Hôpital Foch

${ }^{17} \mathrm{CH}$ de Béthune

${ }^{18} \mathrm{CHU}$ Besancon

${ }^{19} \mathrm{CHU}$ Poitiers

${ }^{20}$ Groupe Hospitalier Diaconesses Croix Saint-Simon Site Avron

${ }^{21}$ CHI Poissy-Saint-Germain-en-Laye Pôle femme-mère-enfant

April 2, 2021

\begin{abstract}
Objective: To assess the incidence of serious complications and reoperations for recurrence after pelvic organ prolapse (POP) surgery and compare the three most common types of repair. Design: Prospective cohort study using a registry. Setting: 19 surgical centres in France. Population: 2309 women participated between 2017 and 2019. Methods: a multivariate analysis including an inverse probability of treatment weighting approach was used to obtain three comparable groups. Main outcome measures: Serious complications and subsequent reoperations for POP recurrence Results: Mean follow-up was 16.6 months. Surgeries included in the analysis were native tissue vaginal repair $(\mathrm{N}=504)$, transvaginal mesh placement (692), and laparoscopic
\end{abstract}


sacropexy with mesh (1113). Serious complications occurred among 52 women (2.3\%), and reoperation for recurrence was required for $32(1.4 \%)$. At one year, the cumulative weighted incidence of serious complications was $1.8 \%$ for native tissue vaginal repair (95\% confidence interval 0-3.9), 3.9\% for transvaginal mesh (2.0-5.9), and 2.2\% for sacropexy (1.1-2.6). Compared with the native tissue vaginal repair group, the risk of serious complications was higher in the transvaginal mesh group (weighted-HR $3.84,2.43-6.08)$, and the sacropexy group $(2.48,1.45-4.23)$, while the risk of reoperation for prolapse recurrence was reduced in both groups (transvaginal mesh [0.22, 0.13-0.39] and sacropexy [0.29, 0.18-0.47]). Conclusions: Laparoscopic sacropexy with mesh appears to have a better risk profile (few serious complications and few reoperations for recurrence) than transvaginal mesh placement (more serious complications) and native tissue vaginal repair (more reoperations for recurrence). These results are useful for informing women and for shared decision making.

Title

Serious complications and recurrences after pelvic organ prolapse surgery for 2309 women in the VIGI-MESH registry

Authors

Xavier Fritel, MD, PhD, 1, 2

Renaud de Tayrac, MD, PhD, 3

Joe de Keizer, MSc, 2

Sandrine Campagne-Loiseau, MD, 4

Michel Cosson, MD, PhD, 5

Philippe Ferry, MD, 6

Xavier Deffieux, MD, PhD, 7

Jean-Philippe Lucot, MD, 8

Laurent Wagner, MD, 9

Philippe Debodinance, MD, 10

Christian Saussine, MD, PhD, 11

Anne-Cécile Pizzoferrato, MD, PhD, 12

Caroline Carlier-Guérin, MD, 13

Thibault Thubert, MD, PhD, 14

Laure Panel, MD, 15

Pierre-Olivier Bosset, MD, 16

Elvis Nkounkou, MD, 17

Rajeev Ramanah, MD, PhD, 18

Thomas Boisramé, MD, 19

Thomas Charles, MD, 20

Cyril Raiffort, MD, 21

Amélie Charvériat, MD, MSc, 1

Stéphanie Ragot, MD, PhD, 2

Arnaud Fauconnier, MD, PhD, 22 


\section{Institutions}

1. Service de Gynécologie, CHU de Poitiers, Poitiers, France

2. Université de Poitiers, INSERM CIC 1402, Poitiers, France

3. Service de Gynécologie, CHU Carémeau, Nîmes, France

4. Service de Gynécologie, CHU Estaing, Clermont-Ferrand, France

5. Service de Gynécologie, CHU de Lille, France

6. Service de Gynécologie, CH de La Rochelle, La Rochelle, France

7. Service de Gynécologie, APHP Antoine-Béclère, Clamart, France

8. Service de Gynécologie, Hôpital Saint-Vincent-de-Paul, Lille, France

9. Service d'Urologie, CHU Carémeau, Nîmes, France

10. Service de Gynécologie, CH de Dunkerque, Dunkerque, France

11. Service d'Urologie, CHU de Strasbourg, Strasbourg, France

12. Service de Gynécologie, CHU de Caen, France

13. Service de Gynécologie, CH de Châtellerault, Châtellerault, France

14. Service de Gynécologie, CHU de Nantes, Nantes, France

15. Service de Gynécologie, Clinique Beau-Soleil, Montpellier, France

16. Service d'Urologie, Hôpital Foch, Suresnes, France

17. Service de Gynécologie, CH de Béthune, Béthune, France

18. Service de Gynécologie, CHU de Besançon, Besançon, France

19. Service de Gynécologie, CHU de Strasbourg, Strasbourg, France

20. Service d'Urologie, CHU de Poitiers, Poitiers, France

21. Service de Gynécologie, Groupe Hospitalier Diaconesses-Croix-Saint-Simon, Paris, France

22. Service de Gynécologie, CHI Poissy-Saint-Germain, Poissy, France

Correspondence:

Prof. Xavier Fritel, Service de Gynécologie-Obstétrique et Médecine de la Reproduction, 2 rue de la Milétrie, CHU de Poitiers, F-86000 Poitiers, France. +33 549443 360.xavier.fritel@univ-poitiers.fr

Running title: Complications after 2309 prolapse surgeries.

Manuscript word count : 2409

Abstract

Objective: To assess the incidence of serious complications and reoperations for recurrence after pelvic organ prolapse (POP) surgery and compare the three most common types of repair.

Design: Prospective cohort study using a registry.

Setting: 19 surgical centres in France.

Population: 2309 women participated between 2017 and 2019.

Methods: a multivariate analysis including an inverse probability of treatment weighting approach was used to obtain three comparable groups.

Main outcome measures: Serious complications and subsequent reoperations for POP recurrence

Results: Mean follow-up was 16.6 months. Surgeries included in the analysis were native tissue vaginal repair $(\mathrm{N}=504)$, transvaginal mesh placement (692), and laparoscopic sacropexy with mesh (1113). Serious complications occurred among 52 women (2.3\%), and reoperation for recurrence was required for $32(1.4 \%)$. At one year, the cumulative weighted incidence of serious complications was $1.8 \%$ for native tissue vaginal repair (95\% confidence interval 0-3.9), 3.9\% for transvaginal mesh (2.0-5.9), and 2.2\% for sacropexy (1.1-2.6). Compared with the native tissue vaginal repair group, the risk of serious complications was higher in the 
transvaginal mesh group (weighted-HR 3.84, 2.43-6.08), and the sacropexy group (2.48, 1.45-4.23), while the risk of reoperation for prolapse recurrence was reduced in both groups (transvaginal mesh [0.22, 0.13-0.39] and sacropexy $[0.29,0.18-0.47])$.

Conclusions: Laparoscopic sacropexy with mesh appears to have a better risk profile (few serious complications and few reoperations for recurrence) than transvaginal mesh placement (more serious complications) and native tissue vaginal repair (more reoperations for recurrence). These results are useful for informing women and for shared decision making.

Keywords: registry, longitudinal study, mesh, pelvic organ prolapse, surgical complication.

Tweetable abstract: Laparoscopic sacropexy showed fewer serious complications than transvaginal mesh and fewer reoperations for recurrence than vaginal repair.

\section{Introduction}

Pelvic organ prolapse is a frequent disability that leads to surgical repair for around one fifth of women.11Smith FJ, Holman CD, Moorin RE, Tsokos N. Lifetime Risk of undergoing surgery for pelvic organ prolapse. Obstet Gynecol 2010;116:1096-100. About 1.1 woman per 1000 undergoes surgery for this condition in France, and around 3.6-3.8 per 1000 aged 60-79 years in U.S.22Subramanian D, Szwarcensztein K, Mauskopf JA, Slack MC. Rate, type, and cost of pelvic organ prolapse surgery in Germany, France, and England. Eur J Obstet Gynecol Reprod Biol 2009;144:177-81.33Wu JM, MD, Matthews CA, Conover MM, Pate V, Funk MJ. Lifetime Risk of Stress Incontinence or Pelvic Organ Prolapse Surgery. Obstet Gynecol 2014;123:1201-6. Information about the risks of adverse effects is essential for choosing the procedure most appropriate to the woman's clinical situation and expectations. To promote shared decision-making, this information must include the frequent or serious complications.44Barber MD. Mesh use in surgery for pelvic organ prolapse, Despite many advances, outcomes after surgery remain far from perfect. BMJ 2015;350:h2910

The information that surgeons provide before the intervention comes from their own experience and their knowledge of the clinical studies. The limited number of patients likely to be covered by both experience and knowledge prevents any exhaustive collection of rare events. Surgical trials often include selected and small samples. Subjects included in trials are often younger and at lower risk than their target population.55Hofmann R, James SK, Jernberg T, Lindahl B, Erlinge D, Witt N, Arefalk G, Frick M, Alfredsson J, Nilsson L, Ravn-Fischer A, Omerovic E, Kellerth T, Sparv D, Ekelund U, Linder R, Ekström M, Lauermann J, Haaga U, Pernow J, Östlund O, Herlitz J, Svensson L; DETO2X-SWEDEHEART Investigators. Oxygen Therapy in Suspected Acute Myocardial Infarction. N Engl J Med 2017;377:1240-9.66Lindsay WA, Murphy MM, Almghairbi DS, Moppett IK. Age, sex, race and ethnicity representativeness of randomised controlled trials in peri-operative medicine. Anaesthesia 2020;75:809-15. This situation should encourage efforts to verify the results of trials in current clinical practice with prospective registries.77Rimmer A. Vaginal mesh procedures need compulsory register, says royal college. BMJ 2018;360:k586.'88Fritel X. Evidence about surgical revision for MUS complications will come from large retrospective cohorts and prospective registers. BJOG 2020;127:1034.

The VIGI-MESH registry enables an exhaustive collection of the operations performed in current clinical practice to treat pelvic organ prolapse and the follow-up of both serious complications and reoperations for recurrence.99Fritel X, Campagne-Loiseau S, Cosson M, Ferry P, Saussine C, Lucot JP, Salet-Lizee D, Barussaud ML, Boisramé T, Carlier-Guérin C, Charles T, Debodinance P, Deffieux X, Pizzoferrato AC, Curinier S, Ragot S, Ringa V, de Tayrac R, Fauconnier A. Complications after pelvic floor repair surgery (with and without mesh): short-term incidence after 1873 inclusions in the French VIGI-MESH registry. BJOG 2020;127:88-97. Now that the registry has been in operation for three years, we report here its medium-term results. We anticipate that the incidence of serious complications and of reoperations for recurrence might differ by the type of surgical repair planned (native tissue vaginal repair, transvaginal mesh placement, or laparoscopic sacropexy with mesh). The objective of our analysis was to assess the risk ratios 
of the different surgical options used in real-world practice for prolapse repair.

\section{Methods}

Participation in the VIGI-MESH registry began after its approval by the Comité de Protection des Personnes Ouest III (Institutional Review Board) in February 2017 (IDRBC 2017-A000308-45), and the posting of the study on Clinicaltrial.gov (NCT03052985). The Agence Nationale de Sécurité du Médicament et des produits de santé (national medicines agency) provided funding for the VIGI-MESH registry, but played no role in data collection or analysis, assessment of the complications, or interpretation of the results. The study had no support or involvement by any manufacturer of mesh.

Participation was offered to all women undergoing surgery for anterior, apical (uterine or vaginal vault) or posterior vaginal prolapse. Each participating woman received information about the VIGI-MESH registry and consented. Surgeons described each operation on a specific case report form. We checked the data collection by reviewing mesh deliveries from the hospital pharmacies and the surgical codes of eligible surgeries recorded by each hospital's medical data department. ${ }^{9}$

This analysis considered three surgical groups: vaginal repair procedure involving native tissue and no mesh (hereafter, vaginal repair), transvaginal placement of mesh (hereafter, transvaginal mesh), and laparoscopic sacropexy, which included colposacropexies (in cases of previous or associated hysterectomy) or colpohysterosacropexies (when the uterus was left in place) that placed mesh by laparoscopy. These procedures are those most frequently used in high-resource countries.11Haya N, Baessler K, Christmann-Schmid C, de Tayrac R, Dietz V, Guldberg R, Mascarenhas T, Nusller E, Ballard E, Ankardal M, Boudemaghe T, Wu JM, Maher CF. Prolapse and continence surgery in countries of the Organization for Economic Cooperation and Development in 2012. Am J Obstet Gynecol 2015;212:755.e1-27 The other, rarer surgical procedures (sacropexy by laparotomy, laparoscopy without mesh, and lateral suspensions) were not included in this analysis as they were rare in our registry. ${ }^{9}$ The planned surgical group was used for the intention-to-treat analysis; for example, a laparoscopic sacropexy converted to a transvaginal mesh procedure was analysed as laparoscopic sacropexy.

\section{Outcomes:}

In accordance with the design of the registry, the surgeons reported complications and reoperations on a specific form during follow-up. To ensure the completeness of the surgeon's reports (for complications and reoperations), we checked the data collected by each hospital's data department to link and monitor medical events after the index surgery and surveyed the participating women. ${ }^{9}$ We analysed the medical data collection as they were received. Queries to surgeons asked them to confirm and detail any serious complications or reoperations when data for these appeared to be missing.

We used the Clavien-Dindo classification to define serious complications: cancellation of planned mesh repair due to intraoperative injury or subsequent surgical intervention related to complication (Grade III), lifethreatening complication (Grade IV), or woman's death (Grade V).11Dindo D, Demartines N, Clavien PA. Classification of surgical complications. Ann Surg 2004;240:205-13. Minor adaptations of the classification designated to describe specific POP-surgery complications were those previously used in the PROSPERE trial.22Lucot JP, Cosson M, Bader G, Debodinance P, Akladios C, Salet-Lizée D, Delporte P, Savary D, Ferry P, Deffieux X, Campagne-Loiseau S, de Tayrac R, Blanc S, Fournet S, Wattiez A, Villet R, Ravit M, Jacquetin B, Fritel X, Fauconnier A. Safety of Vaginal Mesh Surgery Versus Laparoscopic Mesh Sacropexy for Cystocele Repair: Results of the Prosthetic Pelvic Floor Repair Randomized Controlled Trial. Eur Urol 2018;74:167-76. Conversion (for example, from laparoscopy to the vaginal route) due to operative difficulties, such as adhesions, was not considered a complication.33Twijnstra AR, Blikkendaal MD, van Zwet EW, Jansen FW. Clinical relevance of conversion rate and its evaluation in laparoscopic hysterectomy. J Minim Invasive Gynecol 2013;20:64-72. Reoperation for prolapse recurrence was considered a failure but not a complication. For each complication, the operative files of the index surgery and subsequent procedures were 
reviewed by two of the authors (XF and $\mathrm{AC})$.

\section{Statistical analysis:}

Baseline comparisons of the three surgical groups for women's characteristics used ANOVA tests for continuous and Chi-square tests for categorical variables.

We used a propensity score matching approach with inverse probability of treatment weighting to balance the baseline differences between the surgical groups and limit indication bias.11Robins JM, Hernan MA, Brumback B. Marginal structural models and causal inference in epidemiology. Epidemiology 2000;11:55060'22Lunceford JK, Davidian M. Stratification and weighting via the propensity score in estimation of causal treatment effects: a comparative study. Stat Med 2004;23:2937-60. A multinomial logistic regression was constructed to estimate each women's probability of receiving one of the three types of surgeries given their baseline covariates (i.e., the propensity score). Variables of the propensity score model were prespecified before outcome analyses and included age, body mass index, smoking, diabetes, surgical history (hysterectomy, or surgery for stress urinary incontinence or pelvic organ prolapse), physical status score (ASA), menopausal status, and anatomical defect. Stabilized weights were used to estimate the average treatment effect in the entire population, and the extreme weights were truncated.33Austin PC. The performance of different propensity-score methods for estimating differences in proportions (risk differences or absolute risk reductions) in observational studies. Stat Med 2010;29:2137-48. Balance between treatment populations was evaluated by standardized differences of all baseline covariates, with a threshold of 0.1 used to indicate imbalance. ${ }^{16}$

Survival curves were obtained with the Kaplan-Meier estimator. In the absence of earlier events, we censored events as of December 10, 2019. Two weighted frailty models - one for complications and one for recurrence/reoperations - were used to compare the three surgical groups. The models included a non-parametric estimation of the baseline hazard and a gamma frailty term for the centre effect.44Duchateau L, Janssen P, Lindsey P, Legrand C, Nguti R, Sylvester R. The shared frailty model and the power for heterogeneity tests in multicenter trials. Computational Statistics \& Data Analysis 2002;40:603-20.'55Gutierrez RG. Parametric frailty and shared frailty survival models. The Stata Journal 2002;2:22-44.

All statistical tests were two-sided, a p-value $<.05$ was considered significant. A multiple imputation ( $\mathrm{R}$ mice package) strategy was used to deal with the missing data. All statistical analyses were performed with the $\mathrm{R}$ statistical package version 3.6.1 or later (The $\mathrm{R}$ Foundation for Statistical Computing,https://www.Rproject.org/).

Patients were not involved in the development of the VIGI-MESH registry. No core outcome sets were used.

\section{Results}

Between February 2017 and November 2019, 2309 women underwent a surgical repair for pelvic organ prolapse by 110 surgeons in 19 centres, agreed to participate in the registry and were included in the analysis. We estimate that the surgical procedures included in the analysis represent about $76.6 \%$ of eligible procedures for POP repair performed during the study period in the 19 centres (Table S1).

The vaginal repair group included 504 women, the transvaginal mesh group 692, and the laparoscopic sacropexy group 1113 (including 128 with robotic assistance; Table S2). Eight women in the laparoscopic sacropexy group needed a conversion $(0.7 \%)$ : three times to a laparotomic sacropexy, twice to laparoscopic lateral attachment, twice to transvaginal mesh, and once to vaginal repair. One or more other surgical procedures were associated with prolapse surgery, including midurethral sling placement or hysterectomy (Table S2).

The surgical groups differed in terms of age, body mass index, diabetes, menopausal status, smoking, previous hysterectomy, previous surgery for stress urinary incontinence or for pelvic organ prolapse, and anatomical defect (Table S3). Seven of the 12 covariates in the planned propensity score had weighted maximum 
standardized differences below 10\%, while 5 (age, menopausal status, history of POP surgery, history of hysterectomy, and anatomical anterior defect) exceeded the threshold by a maximum of $4 \%$ (Figure S1).

Mean follow-up was 16.6 months (0.4 to 33.8). During this period, 52 women had Clavien-Dindo grade III or higher complications (Table S4). During surgery or in the first 48 hours after: 7 women had an intraoperative injury, 4 a postoperative haemorrhage or hematoma, and 1 a cardiac infarct. From 2 days to 2 months 18 women required a surgical treatment of complications (some women with more than one type of complication): 1 had a peritonitis related to ileal injury, 1 an appendicitis, 1 an incisional eventration with intestinal inclusion after laparoscopy, 1 a bladder retention related to transvaginal mesh, 7 a haemorrhage or hematoma, 4 an ureteral obstruction, 4 a pelvic abscess, 1 a severe postoperative pain after sacrospinofixation, and 2 a vaginal mesh exposure. Between 2 and 12 postoperative months, 20 women required surgical treatment for a complication ( 5 women had 2 complications each): 11 had a vaginal mesh exposure, 1 a bladder mesh exposure after sacropexy, 3 a severe chronic pain, 2 a ureteral obstruction after transvaginal mesh, 2 an incisional hernia after laparoscopy, and 3 a vaginal granuloma. Two women returned to the operating room more than a year after the prolapse surgery: 1 for an incisional hernia after laparoscopy, and 1 for toxin injection for de novo overactive bladder. Complications necessitated 16 interventions to remove the mesh totally or partially $(0.9 \%)$.

The cumulative weighted incidence of serious complications (Figure 1, Table 1) showed a difference between the groups in favour of vaginal repair: $1.8 \%$ for vaginal repair (95\% CI 0-3.9), 3.9\% for transvaginal mesh (2.0-5.9), and $2.2 \%$ for sacropexy (1.1-2.6) at 12 months. Compared with the sacropexy group, the risk of serious complications was higher among women in the transvaginal mesh group (weighted-adjusted HR 1.55, 1.03 to 2.34). A concomitant total hysterectomy was associated with a higher risk of complications (Table $1)$.

Due to a recurrence of the prolapse, a second intervention was required for 32 women (1.4\%): 14 after vaginal repair $(2.8 \%), 6$ after transvaginal mesh $(0.9 \%)$, and 12 after sacropexy (1.1\%). The cumulative weighted incidence of reoperation for prolapse recurrence at 12 months was $1.5 \%$ for vaginal repair (95\%CI 0.4-2.5), $0.7 \%$ for transvaginal mesh (0-1.4), and $1.1 \%$ for sacropexy (0.3-1.9). Compared with the vaginal repair group, the risk of reoperation for prolapse recurrence was reduced in the transvaginal mesh and sacropexy groups (Figure 2; Table 2), with no significant differences between the latter two groups.

Postoperative stress incontinence resulted in reoperation for 61 women (2.6\%): 6 after vaginal repair (1.2\%), 28 after transvaginal mesh (4.0\%), and 27 after sacropexy $(2.4 \%)$.

In accordance with the design of the registry, we audited a sample of women included in 2017 and 2018 (61\% of 1,575 women responded). The responses showed that $96.3 \%$ of the serious complications were already listed in the registry as were $94.1 \%$ of the reoperations for prolapse recurrence.

\section{Discussion}

We report an analysis of data collected from routine care to compare the medium-term efficacy and safety of the 3 most common surgical procedures for pelvic organ prolapse repair in a population of 2309 women. The events were uncommon: the rate of serious complications ranged from $1.4 \%$ after native tissue vaginal repair to $3.8 \%$ after transvaginal mesh placement, and the rate of reoperations for recurrence from $2.8 \%$ after vaginal repair to $0.9 \%$ after transvaginal mesh. The women in the vaginal repair group were exposed to the lowest risk of serious complications and the highest risk of reoperation for recurrence, the women in the transvaginal mesh group to the highest risk of serious complications and the lowest risk of reoperation for recurrence, and the women in the laparoscopic sacropexy group to an intermediate risk of serious complications and a low risk of reoperation for recurrence, like in the transvaginal mesh group.

The strengths of our study are its large prospective registry including 19 centres and numerous surgeons; these features enable the detection of rare events. The analysis covers operations performed in real-life situations: complex clinical situations were not excluded. Indeed, a high proportion of our population would not have 
been included in a randomized trial, because they were too old, because of prolapse recurrence after previous surgery, or various comorbidities. The regular and routine verification of the information from the hospitals databases and from a sample of the participants is evidence of validity.

Our primary outcome was based on a robust criterion, as the modified Clavien-Dindo classification has been found to be a valid and reproducible classification of complications in various surgical domains. ${ }^{11,12}$ It is easy to verify serious complications and reoperations by consulting the hospital's data department and the women who had surgery.

Our results must be interpreted considering the absence of randomization. The surgical groups had different characteristics. However, we took the women's preoperative characteristics into account with the propensity score to emulate a comparative trial. ${ }^{14}$ The data about the construction of the propensity score appear reassuring regarding potential unmeasured confounders that may bias our results. In addition, within each surgical group, there were multiple and different surgical procedures. These limitations are the price of a "real world" study design, which can introduce variance and limit statistical power.

Another limitation is the medium-term follow-up averaging 16 months. However, a Finnish cohort found that most complications of POP surgery occurred within the two first postoperative months.11Wihersaari O, Karjalainen P, Tolppanen A-M, Mattsson N, Jalkanen J, Nieminen K. Complications of Pelvic Organ Prolapse Surgery in the 2015 Finnish Pelvic Organ Prolapse Surgery Survey Study. Obstet Gynecol 2020;136:1135-44. Five year after transvaginal mesh placement, another study found that $79 \%$ of the mesh exposures occurred during the first postoperative year.22Jacquetin B, Hinoul P, Gauld J, Fatton B, Rosenthal C, Clavé H, Garbin O, Berrocal J, Villet R, Salet-Lizée D, Debodinance P, Cosson M. Total transvaginal mesh (TVM) technique for treatment of pelvic organ prolapse: a 5-year prospective follow-up study. Int Urogynecol $\mathrm{J}$ 2013;24:1679-86.

Our results about the relative risk of serious complications are similar to earlier comparative studies, reporting fewer complications with vaginal surgery without mesh.33Siddiqui NY, Cara L. Grimes CL, Casiano ER, Abed HT, Jeppson PC, Olivera CK, Sanses TV, Steinberg AC, South MM, Balk EM, Sung VW. Mesh Sacrocolpopexy Compared With Native Tissue Vaginal Repair, A Systematic Review and Meta-analysis. Obstet Gynecol 2015;125:44-55.'44Maher C, Feiner B, Baessler K, Christmann-Schmid C, Haya N, Brown J. Surgery for women with anterior compartment prolapse. Cochrane Database of Systematic Reviews 2016, Issue 11. Art. No.: CD004014. The incidence of complications in our registry was lower than that reported in several trials. This difference may be explained by the definition used for complications, which considered only serious Clavien-Dindo complications. The risk of reporting failure seems marginal, as we systematically and regularly verified the information with the hospitals' data departments and with the women. Most participating hospitals are teaching hospital centres specialized in management of recurrent prolapse. This point may explain the low complication rate. Verification of this hypothesis might also mean that transvaginal mesh use by experienced centres might be at low risk of complications.

Today as concerns about mesh safety abound, our results offer real-world information for women that can enable them to participate in the choice of technique most appropriate for them. It suggests that in the medium term, laparoscopic sacropexy may have the best risk profile. Vaginal repair has a minimal risk of serious complications, but a higher risk of reoperation for recurrence.

Because reporting the specific functional results of prolapse surgery for each type of surgical procedure appears useful, we have planned a questionnaire to send to all the women in our registry. Identifying the technical surgical details that are at greater risk of complications than others (such as the type of mesh or the sutures used) should help improve surgical procedures. Assessing the effect of the experience of both surgeons and centres could be useful for determining if some procedures should be reserved to expert centres.

\section{Disclosure of interests}

All authors completed the ICMJE uniform disclosure form. XF, JK, SCL, PF, JPL, LW, PD, CS, ACP, 
CCG, TT, LP, POB, EN, RR, TB, TC, CR, AC, SR, and AF have nothing to disclose. Prof. Renaud de Tayrac reports grants, personal fees or other from Boston Scientific and Coloplast, during the conduct of the study; non-financial support from Wellspect, outside the submitted work. Prof. Michel Cosson reports personal fees from Boston Scientific, Coloplast, Promedon, and AMI, outside the submitted work. Prof. Xavier Deffieux reports personal fees or other from Urgo-Tech, Coloplast, Allergan, Laborie, Hologic, Sanofi, and Nanobiotics, outside the submitted work.

\section{Contribution to authorship}

Study concept and design: Fritel, Fauconnier, Ragot.

Acquisition of data: Fritel, de Tayrac, Campagne-Loiseau, Cosson, Ferry, Hummel, Deffieux, Lucot, Wagner, Debodinance, Saussine, Pizzoferrato, Carlier-Guérin, Thubert, Panel, Bosset, Nkounkou, Ramanah, Boisramé, Charles, Bressler, Charvériat, Raiffort, Fauconnier.

Analysis and interpretation of data: Fritel, Fauconnier, de Keizer, Ragot.

Drafting of the manuscript: Fritel, Fauconnier, Ragot.

Statistical analysis: Fritel, de Keizer, Ragot.

Obtaining funding: Fritel.

Xavier Fritel had full access to all the data in the study and takes responsibility for the integrity of the data and the accuracy of the data analysis. He attests that all listed authors meet authorship criteria and that no others meeting the criteria have been omitted.

We affirm that our manuscript is an honest, accurate and transparent report of the VIGI-MESH registry; that no important aspects of the registry have been omitted; and that any discrepancies from the registry as originally planned and registered have been explained.

Details of ethics approval

Our study complies with French law. The Institutional Review Board (Comité de Protection des Personnes Ouest III) approved the protocol on 9 February 2017 (IDRBC 2017-A000308-45), and the study was registered by the national data protection authority (Commission Nationale Informatique et Libertes, CNIL) on 16 August 2017 (DR-2017-245).

\section{Funding}

The national medicines agency (Agence Nationale de Sécurité du Médicament et des produits de santé, ANSM) provided the funding for the study, but played no role in data collection or analysis, assessment of the complications, or interpretation of the results.

Acknowledgements:

We thank Lucie Merlet (CHU de Poitiers), Aurélie Comptour (CHU Carémeau), Florence Tartarin (CHU de Poitiers), Elodie Migault (CHU de Poitiers), and Pierre-Jean Saulnier (Université de Poitiers) for their support or contribution to collecting and cleaning the data. We thank Jo Ann Cahn for the editing (CHU de Poitiers).

We thank 110 surgeons of the VIGI-MESH Group who included into the registry: Adnan Aboukassem, Chérif Akladios, Emmanuelle Arsène, Jean-Sébastien Aucouturier, Georges Bader, Emmanuel Bailly, JeanJacques Baldauf, Stéphanie Bartolo, Marie-Line Barussaud, Fanny Béchard, Simon Bernardeau, Clément Biscans, Thomas Boisramé, Deepak Boodhun, Pierre-Olivier Bosset, Revaz Botchorishvili, Michel Boukaram, Aude Brams, Laurent Bressler, Clément Bruhat, Sandrine Campagne-Loiseau, Michel Canis, Caroline Carlier-Guérin, Victor Cavillon, Olivier Celhay, Thomas Charles, Amélie Charvériat, Armand Chevrot, Pierre Collinet, Arnaud Cornille, Michel Cosson, Pierre Costa, Christophe Courtieu, Laurent Courtois, Sandra Curinier, Eric Darnis, Renaud de Tayrac, Philippe Debodinance, Xavier Deffieux, Pierre-Olivier Delpech, 
Véronique Delporte, Anne Dubois, Emilie Faller, Brigitte Fatton, Arnaud Fauconnier, Philippe Ferry, Cécile Féyeux, Xavier Fritel, Victor Gabriele, Pierre Gadonneix, Olivier Garbin, Florent Genty, Géraldine Giraudet, Pascale Gres, Pauline Gueudry, Jean-François Haab, Audrey Hedde, Aline Host, Michel Hummel, Estelle Jean dit Gautier, Aminata Kane, Sophie Le Gouic, Isabelle Le Teuff, Gil Lebreton, Lise Lecointre, Grégoire Léon, Jean-Philippe Lucot, Yolande Maisonnette, Lucile Martin, Aurore Marx, Pascal Mouracade, Elvis Nkounkou, Corinne Palamara, Laure Panel, Petit Nicolas, Caroline Pettenati, Laurence Peyrat, Pierre Pillot, Anne-Cécile Pizzoferrato, Jean-Luc Pouly, Clothilde Poupon, Michel Prudhomme, Benoît Rabishong, Rajeev Ramanah, Hélène Ricard, Jérémie Ripoche, Géraldine Rivaux, Jennifer Salerno, Delphine Salet-Lizée, Richard Sarfati, Maxence Sarradin, Christian Saussine, Elodie Schuller, An Segaert, François Stoll, Yannick Thirouard, Thibault Thubert, Caroline Trichot, Mélusine Turck, David Vandendriessche, Edouard Vaucel, Sarah Vieillefosse, Anne Villot, Denis Vinatier, Etienne Vincens, Marie Vinchant, Béatrice Vinson-Bonnet, Laurent Wagner, Soraya Wapler, Sophie Warembourg.

\section{References}

Figure and table legends

Figure 1. Kaplan-Meier curve free of serious complication (Clavien-Dindo grade III or more) as a function of time (months) and of surgical group (2309 women).

Figure 2. Kaplan-Meier curve free of reoperation for prolapse recurrence as a function of time (months) and of surgical group (2309 women).

Table 1. Risk factors for serious complication (Clavien-Dindo grade III or more). Frailty model with centre as a random effect $(\mathrm{N}=2309)$

Table 2. Risk factors for reoperation for prolapse recurrence. Frailty model with center as a random effect $(\mathrm{N}=2309)$

Online supporting information

Figure S1. Standardized differences between surgical groups before and after adjustment

Table S1. Detail of inclusions in each centre.

Table S2. Surgical procedures for pelvic organ prolapse $(\mathrm{N}=2309)$.

Table S3. Women's characteristics at the time of the index surgery for pelvic organ prolapse $(\mathrm{N}=2309)$. Comparison between surgical groups with ANOVA tests for continuous and Chi-square tests for categorical variables.

Table S4. Description of serious complications (Clavien-Dindo Grade III or more) among 52 women by type, time to revision after POP surgery, and type of care for complication (some women may have more than one type of complication).

\section{Hosted file}

Tables \& Figures.pdf available at https://authorea.com/users/405406/articles/516404-seriouscomplications-and-recurrences-after-pelvic-organ-prolapse-surgery-for-2309-women-in-thevigi-mesh-registry 